\title{
Correlation of the Religious and the Paranormal Beliefs to Personality
}

\author{
Dr. Malik Roshan Ara ${ }^{1}$
}

\section{ABSTRACT}

Beliefs happen to be the most significant indicators of a person's overall personality and provide us a window on his mental constructs. The study of the paranormal activities and phenomena has been riddled with controversy since its conception and previous researches revealed mixed and contradictory findings. The present study focuses to study correlation between Religious beliefs, paranormal beliefs and the personality factors across different religious groups, among individuals of different age groups and socio-economic status. Significant positive correlation was found between religiosity and paranormal beliefs as well as between religiosity and the traditional religious beliefs, psi and witchcraft subscales of the paranormal beliefs scale. Results also report a negative correlation between religious beliefs and the spiritualism, extraordinarylife-forms and precognition subscales of the paranormal beliefs scale. Neuroticism was found to be the only personality factor significantly predicting paranormal beliefs. Muslims scored higher on the traditional religious beliefs subscale in comparison with the Hindus. On the other hand, among the Hindus a higher degree of endorsement of witch-craft was observed. However, so far as the Christians are concerned no significant differences were observed either on global paranormal beliefs or any of the subscales of the paranormal beliefs. Socially marginal groups were found to be more susceptible to paranormal beliefs. They scored higher on psi, witchcraft, superstition, spiritualism, precognition as well as the total- paranormal beliefs scale.

Keywords: Religious Beliefs, Paranormal Beliefs, Personality Factors.

Beliefs happen to be the most significant indicators of a person's overall personality and provide us a window on his mental constructs. Yet there is little agreement as to what they are; or how they should be construed. They are basic to our understanding of a wide range of central phenomena in modern psychology. Beliefs are the key components of our personalities and sense of identity, and our expression of beliefs often defines us to others. The topic of beliefs is vast and diverse. It crosses many disciplinary boundaries both within psychology and across many traditional disciplines. It has an extensive coverage ranging from traditional beliefs to

${ }^{1}$ Department of school education Government of J\&K

(C) 2015 I M Ara; licensee IJIP. This is an Open Access Research distributed under the terms of the Creative Commons Attribution License (http://creativecommons.org/licenses/by/2.0), which permits unrestricted use, distribution, and reproduction in any Medium, provided the original work is properly cited. 


\section{Correlation of the Religious and the Paranormal Beliefs to Personality}

anomalous or paranormal beliefs. While on one hand a great deal of research has been carried out on traditional beliefs and they have been discussed at length, anomalous beliefs, on the other hand, are the beliefs in powers and phenomena that lie outside the realm of normal science; and as such this field has been generally left under-investigated. Anomalous or paranormal beliefs account for the emphasis on Extrasensory perception (ESP), astrology, witchcraft, ghosts, unidentified flying objects (UFO) abductions and the like. In order to account for people's real experiences in these areas, one has to deal with the topics about which much has been read and heard, but haven't had an easy relationship with mainstream psychology.

Nearly every culture throughout history has reported beliefs and experiences with paranormal phenomena. Paranormal phenomena are defined as events that violate the boundaries of current scientific belief. In spite of having no definitive proof to substantiate these beliefs more than 90 percent of American adults profess to believe in at least one phenomenon (Gallup, 1997). Research indicates that the rise in paranormal phenomena beliefs began in the 1960's with the massive sale of parapsychology books. Ouija boards were outselling monopoly games (Truzzi, 1972). Over the last three decades or so, scholars from a spectrum of disciplines have lent their expertise in trying to understand the increasing beliefs in paranormal phenomena. Despite the fact that thousands of people are reporting paranormal events each year, the majority of research is in the form of public opinion polls, that encompass a yes/no answer format, leaving the reader to fill in the rest.

The study of the paranormal activities and phenomena has been riddled with controversy since its conception. It is claimed that some people, utilizing sense beyond the ordinary, exhibit powers that cannot be explained by traditional science. Many wondrous or anomalous experiences support belief in spiritual powers or forces; and as such, play an important role in the development of religious ideologies. Scientific interest in the subject is of a relatively recent origin, but belief in the reality of such phenomena has been widespread since the earliest recorded times. Before the rise of modern science, the causation of all the complex phenomena was very proudly understood and hence appeals to non-material agencies (ghosts, sorcerers, demons and mythological beings) took the place of a causal scientific explanation. In order to explain why people believe in "unbelievable" things, one must first ascertain what the levels of belief are. Results from a United States survey, conducted by Schmeidler (1985) indicated that 51percent of respondents reported an experience with ESP. A poll conducted in 1990, stated that 49 percent of the respondents believed in ESP, 38 percent believed in ghosts, and 33 percent believed in aliens (Newport \& Strausberg 2001). Another study conducted in 1997, supports these findings and shows that over 50 percent of respondents indicated a belief in ghosts, while one-third responded they have had an experience with extrasensory perception (ESP) (Sparks, Glenn, Nelson, \& Leigh, 1997). Another survey revealed that 59 percent of respondents were believers in the paranormal (Blackmore, 1997). Another study found that 49 percent of the participants believed that the extraterrestrial life exists, and 33 percent believed UFOs exist (Biasco \& Nunn, 2000). Patry and Pelletier (2001) found that when participants were asked whether they personally knew someone who had allegedly seen a UFO, 24 percent answered yes, 


\section{Correlation of the Religious and the Paranormal Beliefs to Personality}

and 48 percent believed UFOs were real, as opposed to 35 percent who did not. Patry and Pelletier also found men were significantly more likely to believe in UFOs than females. Researchers have been searching for evidence that may distinguish believers of paranormal phenomena from non-believers. One area of great interest is the possible relationship between religiosity/ spirituality and belief in paranormal phenomena. Numerous researchers have found that religious preference (Fox 1992) and religious orientation (MacDonald 1992) are not correlated to reported paranormal experiences. A study in (2001) investigated the correlation between religiosity and belief in paranormal phenomena, researchers found that religiosity was not significantly correlated with belief in the paranormal (Beck \& Miller, 2001). However, Beck \& Miller found that experiences of negative affect over the preceding year were positively correlated with belief in the paranormal. (Sparks, et al).

Numerous researchers have looked toward demographics such as age, sex, and education level to explain differences in beliefs. Studies indicate that there is a significant difference in beliefs in the paranormal between genders ( Irwin,1993). Females have expressed stronger beliefs in the paranormal than males. Females were higher in their beliefs of ESP, and ghosts, while men had higher beliefs in UFOs and the Loch Ness Monster (Irwin, 1993). Blackmore (1997) found that there was a large significant sex difference between believers and non-believers. Blackmore found that over 70 percent of the female respondents were believers, compared to 48 percent of the males. The education level of participants is also considered when investigating belief systems. Henri Broch (2000) reports that a French opinion poll demonstrated a positive relationship between the respondent's education level and their belief in paranormal phenomena. The Princeton Research Association reported survey results that found no significant differences in beliefs in paranormal phenomena between high school dropouts and non-dropouts (Goode, 2002). A Yankelovick poll found no significant differences between education level and belief in UFOs (Goode, 2002). The Pew research center found that approximately 20-30 percent more of the least educated respondents reported beliefs in angels (Goode, 2002).

It is perhaps due to the widespread existence of these beliefs, that the investigation of the correlation of the paranormal and religious beliefs with personality has received considerable attention in the recent years. However, little attention has been paid to study the religious and paranormal phenomena together in relation to personality. Moreover, is has also been observed that most of the studies concerning paranormal beliefs, religiosity and the personality, conducted so far, were being carried out on the student populations. It, therefore, seems relevant here to study the personality correlates of religiosity and paranormal beliefs among the adults and the elderly among whom these two factors are expected to be highly endorsed. It would also be relevant to take into account certain other demographic variables, such as, age, socio-economic status and the level of education for a more informed research into the personality correlates of religiosity and paranormal beliefs. 


\section{PURPOSES OF THE INVESTIGATION}

The present study seeks to confirm the literature findings and to extend the boundaries of the previous researches by investigating the relationship between personality, paranormal beliefs and religiosity. In the light of the aims of the study and the previous researches reported, the following hypothesis will be tested:

\section{Hypothesis}

1. There will be some relationship between paranormal beliefs and religious beliefs.

2. Socially marginal groups and those low on education will be more susceptible to paranormal beliefs.

3. Women will endorse greater global paranormal beliefs.

4. There will be a higher degree of endorsement of the paranormal beliefs among the adults and elderly.

5. The personality factors expected to correlate with paranormal beliefs are Neuroticism and extraversion.

6. The personality factors expected to correlate with religiosity are Agreeableness and Conscientiousness.

7. There will be a difference in the degree of belief in paranormal across different religions.

\section{Methodology \\ Participants}

The participants of the present study comprised of 300 people; a hundred each from the three major religious groups' viz. Hindus, Muslims and Christians. The research population consisted of individuals, exclusively of Indian origin and belonged to the states of Uttarpradesh, Bihar and Rajasthan. The selection of the participants as per the requirements of the present study was done randomly from the three major religious groups. The age of the participants ranged from 15 to 71 years, with a mean age of 21.5 years. The participants were categorized into three age groups as, young, adults and elderly, those in the age group of 15- 19 years were categorized as young; those in the age group of 20-40 years were categorized as adults and finally those in the age group of 41 years and above were categorized as elderly. The entire group of participants of the present research was also divided in terms of gender (females and males). Further categorization of the participants was done on the basis of their socio-economic status. The selection of the participants for this particular category was done on the basis of their monthly income and level of education. The low socio-economic status group of participants comprised of individuals whose monthly income was very low (i.e. up to Rs.2000 per month) and they were either illiterate or very low on education (slum-dwellers, rickshaw-pullers or daily-waged employees). So far as the participants of a high socio-economic status are concerned, these included individuals who have had a decent monthly income (i.e. from Rs.10, 000 to 40,000 per month). 


\section{Correlation of the Religious and the Paranormal Beliefs to Personality}

The participants in this category were mostly, school teachers, doctors, bank-employees and University Professors and Lecturers.

\section{Measures}

The following inventories were used to assess the constructs of paranormal beliefs, religious beliefs and personality.

\section{RELIGIOSITY SCALE (Decker and Broota, 1985)}

The Religiosity Scale (Decker and Broota, 1985) was used to measure the extent of an individual's dependency on the supernatural being adherence to the doctrines of one's faith. The final scale consisted of 44 items out of which 25 were positive and 19 were negative. The presence of both positively and negatively worded items is essential, for it avoids the tendency of the respondents to develop a response set, that might occur, were the items are only positive or only negative. The reliability of the final scale was established using the split-half technique. The items of the scale were split into two equivalent forms using the odd-even methods. The reliability co-efficient of the half tests was 0.91 (using Pearson's product moment). The obtained values were corrected for length using Spearman Brown formula and were 0.96. Thus, the reliability co-efficient for the religiosity scale was found to be 0.96 for an adult sample of subjects.

\section{REVISED PARANORMAL BELIEF SCALE (RBPS) (Tobayck, 1988)}

The Revised Paranormal Belief Scale (RPBS), (Tobayck, 1988) was used to measure paranormal beliefs. It is a 26 item self report scale, measuring the following seven forms of paranormal beliefs: traditional religious beliefs, Psi beliefs, witchcraft, superstition, spiritualism, extraordinary life forms and precognition. The extraordinary life forms subscale was modified slightly by replacing two items. Item 6, i.e., the abdominal snowman of Tibet exists, was replaced with: the UFOs exist. Also item 13, i.e., the Loch Ness monster of Scotland exists was replaced with: the extraterrestrial objects exist. Responses to each item are scored on a seven point Likert Scale. The Cronbach-Alpha coefficient for the Revised Paranormal Belief Scale is 0.91 .

\section{NEO-five Factor Inventory (NEO-FFI) (Costa and McCrea, 1992)}

The NEO-FFI (Costa and McCrea, 1992) is a self report measure of personality features that make up an influential model of personality known as Five Factor Model (FFM). The FFM of personality has evolved over the last four decades (Digman, 1990) and has roots in both the lexical tradition (i.e. the analysis of trait adjectives found in English and other languages) as well as the factor analytic tradition in personality research. As operationalised by the NEO-PU-R, the five factors or domains are: Neuroticism, Extraversion, Openness to experience, Agreeableness and Conscientiousness. NEO-FFI is a 60 item version of form-S of the NEO-PI-R that provides a brief, comprehensive measure of the five domains of personality. It consists of five 12 item 
scales that measure each domain. Each of the items of the scale is scored on a five point Likert scale.

\section{Procedure}

The set of questionnaire, which included the three scales measuring paranormal beliefs, religious beliefs and personality factors, was distributed to the participants using the opportunity method. Participants were informed that their responses would remain confidential and would be used exclusively for research purpose. They were also informed that they could choose not to complete the questionnaire, if they wished. In the case of the participants from socially marginal groups, individual items of each of the inventories were explained in there regional languages.

\section{$\underline{\text { Ethics }}$}

Because of the involvement of the human participants in the research process, the ethical principles for conducting research, as stated by the BPS (1992), were closely followed. It was made clear to all the participants both verbally and in writing that participation was entirely voluntary and that they were free to withdraw consent for their completed questionnaires at any time, and for whatever reason.

\section{$\underline{\text { Data analysis }}$}

The responses given by the participants of the present study were systematized and analyzed using appropriate statistical techniques. One way analysis of variance, t-test, product moment coefficient of correlation and multiple regression analysis were used to analyze the data.

\section{RESULTS AND DISCUSSION}

For testing Hypothesis - 1: there will be significant relationship between paranormal beliefs and religious beliefs, Pearson product moment correlation is used as follows: 
Table 1. Pearson Product Moment Correlation Coefficients between Paranormal beliefs including its subscales and Religious beliefs.

\begin{tabular}{|l|l|l|l|l|l|l|l|l|}
\hline \multicolumn{1}{|l|}{ Var 1 } & TRB & PSI & WC & ST & SP & EOLF & PR & Total P \\
\cline { 3 - 8 } & & & & & & & & \\
\hline \multirow{2}{*}{ Religiosity $=0.628^{* * *}$} & $0.199^{* * *}$ & $0.294^{* * *}$ & 0.081 & -0.088 & -0.051 & -0.056 & $0.236^{* *}$ \\
& $\mathrm{P}=0.000$ & 0.001 & 0.000 & 0.159 & 0.127 & 0.380 & 0.335 & 0.000 \\
& $\mathrm{~N}=300$ & 300 & 300 & 300 & 300 & 300 & 300 & 300 \\
\hline
\end{tabular}

** Correlation is significant at 0.01 level.

Note: TRB=Traditional religious beliefs, $\mathrm{PSI}=$ Psycho-kinesis, $\mathrm{WC}=$ Witchcraft, $\mathrm{ST}=$ Superstition, $\mathrm{SP}=$ Spiritualism, EOLF=Extra ordinary life forms, $\mathrm{PR}=$ Precognition, Total $\mathrm{p}=$ Global paranormal beliefs.

Table 1 shows that there is significant correlation between religious beliefs and paranormal beliefs at 0.01 level of significance $(\mathrm{r}=0.236, \mathrm{p}=0.000)$. The table also shows that there is significant correlation between religious beliefs and TRB $(r=0.682, p=0.00)$, Psi $(r=0.199$, $\mathrm{p}=0.001)$ and $\mathrm{WC}(\mathrm{r}=0.294, \mathrm{p}=0.000)$ subscales of the paranormal belief scale.

For testing Hypothesis - 2: The personality factors expected to correlate with paranormal beliefs are Neuroticism and Extraversion, step-wise multiple regression analysis is used as follows:

Table 2. Model summary of the prediction of paranormal beliefs from Personality factors.

\begin{tabular}{|c|c|c|c|}
\hline R & R- Square & Adjusted R-Square & Std.error of estimate \\
\hline 0.184 & 0.034 & 0.031 & 12.62424 \\
\hline
\end{tabular}


Table 3. ANOVA table of a multiple regression analysis for the prediction of Paranormal beliefs from Personality factors.

\begin{tabular}{|c|l|l|l|l|l|l|}
\hline Variable & \multicolumn{2}{|l|}{ Sum of Squares } & Df & Mean square & F & P \\
\hline \multirow{3}{*}{$\mathrm{N}$} & Regression & 1670.248 & 1 & & & \\
& & & & 1670.248 & 10.480 & 0.001 \\
\cline { 2 - 5 } & Residual & 47492.669 & 298 & 159.371 & & \\
\cline { 2 - 5 } & Total & 49162.917 & 299 & & & \\
\hline
\end{tabular}

Table 4: Coefficients of regressions for the prediction of the Paranormal beliefs from Personality factors.

\begin{tabular}{|l|l|l|l|l|l|}
\hline Variable & B & Std.error & B & t & P \\
\hline Constant & 68.196 & 2.791 & & 24.438 & 0.0005 \\
$\mathrm{~N}$ & 0.389 & 0.120 & 0.184 & 3.237 & 0.001 \\
\hline
\end{tabular}

Table 4 reports the results of a multiple regression analysis for the prediction of paranormal beliefs from the personality factors. A multiple regression analysis using the step-wise method was used to predict which one of the five personality subscales, if any are the significant predictors of paranormal beliefs. No significant model emerged for the predictor variables (F 1, $298=10.480, \mathrm{p}>0.05)$.the adjusted $\mathrm{R}$ square $=0.031$. As reported in the table, $\mathrm{N}(\beta=0.184, \mathrm{p}<$ 0.001). Therefore just one out of the five personality subscales is found to be a significant predictor of the paranormal beliefs. Extraversion, Openness, Agreeableness and Conscientiousness were not found to significantly predict paranormal beliefs.

For testing hypothesis - 3: The personality factors expected to correlate with religiosity are Agreeableness and Conscientiousness, step-wise multiple regression analysis is used as follows:

Table 5. Model summary of the prediction of Religiosity from Personality factors.

\begin{tabular}{|l|l|l|l|}
\hline $\mathbf{R}$ & R-Square & R-Square Adjusted & Std. Error of estimate \\
\hline 0.376 & 0.141 & 0.133 & 20.81746 \\
\hline
\end{tabular}


Table 6. ANOVA table of a multiple regression analysis for the prediction of Paranormal beliefs from Personality factors.

\begin{tabular}{|l|l|l|l|l|l|l|}
\hline Variable & \multicolumn{2}{|l|}{ Sum of Squares } & df & $\begin{array}{l}\text { Mean } \\
\text { square }\end{array}$ & F & p \\
\hline \multirow{2}{*}{ O, N \&C } & Regression & 21100.397 & 3 & \multirow{2}{*}{$\begin{array}{l}7033.466 \\
433.367\end{array}$} & 16.230 & \multirow{2}{*}{0.0005} \\
\cline { 2 - 6 } & Residual & 128276.6 & 296 & & \\
\cline { 2 - 6 } & Total & 149377.0 & 299 & & \\
\hline
\end{tabular}

Table 7: Coefficients of regressions for the prediction of the Paranormal beliefs from Personality factors.

\begin{tabular}{|l|l|l|l|l|l|}
\hline Variable & B & Std.error & B & t & P \\
\hline Constant & 157.139 & 10.967 & & 14.329 & 0.0005 \\
$\mathrm{O}$ & -1.513 & 0.277 & -0.294 & -5.464 & 0.0005 \\
$\mathrm{~N}$ & 0.855 & 0.202 & 0.233 & 4.241 & 0.0005 \\
$\mathrm{C}$ & 0.500 & 0.213 & 0.128 & 2.343 & 0.020 \\
\hline
\end{tabular}

Table -7 reports the results of a multiple regression analysis for the prediction of the religious beliefs from the personality factors. A multiple regression analysis using the step-wise method was used to predict which one of the five personality subscales, if any are the significant predictors of religiosity. No significant model emerged for the predictor variables $(\mathrm{F} 3,296=$ $16.230, \mathrm{p}>0.05)$.the adjusted $\mathrm{R}$ square $=0.133$. As reported in the table, $\mathrm{O}(\beta=-0.294, \mathrm{P}<0.001)$, $\mathrm{N}(\beta=0.233, \mathrm{P}<0.001)$ and $\mathrm{C}(\beta=0.128, \mathrm{P}<0.001)$. Therefore just three out of the five personality subscales are significant predictor of religiosity. Extraversion and Agreeableness were not found to significantly predict religious beliefs. Therefore just three out of the five Personality subscales were found to be significant predictors of the religious beliefs. The table also reveals that the $\beta$ value for $O$ (i.e. openness) is negative which means that there exists a significant inverse relation between the two constructs; i.e. high scores on openness subscale indicate a decrease in the religiosity score.

For testing Hypothesis - 4: There will be difference in the degree of endorsement of paranormal beliefs across different religions, one-way analysis of variance is used as follows: 
Table 8: Descriptive statistics of the individuals of three religious groups on Paranormal beliefs and its subscales.

\begin{tabular}{|c|c|c|c|c|c|}
\hline Paranormal beliefs & Groups & $\mathbf{N}$ & Mean & SD & Std.error of mean \\
\hline $\begin{array}{l}\text { Traditional religious } \\
\text { beliefs }\end{array}$ & $\begin{array}{l}\text { Muslim } \\
\text { Hindu } \\
\text { Christian } \\
\text { Total }\end{array}$ & $\begin{array}{l}100 \\
100 \\
100 \\
300\end{array}$ & $\begin{array}{l}17.3700 \\
15.4900 \\
16.4700 \\
16.4433\end{array}$ & $\begin{array}{l}2.49304 \\
2.99324 \\
3.14773 \\
2.98269\end{array}$ & $\begin{array}{l}0.24930 \\
0.29932 \\
0.31477 \\
0.17221\end{array}$ \\
\hline Psi & $\begin{array}{l}\text { Muslim } \\
\text { Hindu } \\
\text { Christian } \\
\text { Total }\end{array}$ & $\begin{array}{l}100 \\
100 \\
100 \\
300\end{array}$ & $\begin{array}{l}11.7500 \\
12.3300 \\
12.5000 \\
12.1933\end{array}$ & $\begin{array}{l}3.02306 \\
3.30917 \\
2.91461 \\
3.09319\end{array}$ & $\begin{array}{l}0.30231 \\
0.33092 \\
0.29146 \\
0.17859\end{array}$ \\
\hline Witchcraft & $\begin{array}{l}\text { Muslim } \\
\text { Hindu } \\
\text { Christian } \\
\text { Total }\end{array}$ & $\begin{array}{l}100 \\
100 \\
100 \\
300\end{array}$ & $\begin{array}{l}11.8900 \\
10.2500 \\
11.3900 \\
11.1767\end{array}$ & $\begin{array}{l}3.74946 \\
3.49133 \\
3.27493 \\
3.56574\end{array}$ & $\begin{array}{l}0.37495 \\
0.34913 \\
0.32749 \\
0.20587\end{array}$ \\
\hline Superstition & $\begin{array}{l}\text { Muslim } \\
\text { Hindu } \\
\text { Christian } \\
\text { Total }\end{array}$ & $\begin{array}{l}100 \\
100 \\
100 \\
300\end{array}$ & $\begin{array}{l}6.1300 \\
6.4400 \\
6.2700 \\
6.2800\end{array}$ & $\begin{array}{l}1.93665 \\
2.67921 \\
2.13605 \\
2.26835\end{array}$ & $\begin{array}{l}0.19366 \\
0.26792 \\
0.21361 \\
0.13096\end{array}$ \\
\hline Spiritualism & $\begin{array}{l}\text { Muslim } \\
\text { Hindu } \\
\text { Christian } \\
\text { Total }\end{array}$ & $\begin{array}{l}100 \\
100 \\
100 \\
300 \\
\end{array}$ & $\begin{array}{l}9.9200 \\
11.0100 \\
10.6300 \\
10.5200 \\
\end{array}$ & $\begin{array}{l}3.57793 \\
3.27369 \\
3.16437 \\
3.36263 \\
\end{array}$ & $\begin{array}{l}0.35779 \\
0.32737 \\
0.31644 \\
0.19414 \\
\end{array}$ \\
\hline $\begin{array}{l}\text { Extraordinary life } \\
\text { forms }\end{array}$ & $\begin{array}{l}\text { Muslim } \\
\text { Hindu } \\
\text { Christian } \\
\text { Total }\end{array}$ & $\begin{array}{l}100 \\
100 \\
100 \\
300\end{array}$ & $\begin{array}{l}8.9500 \\
10.1600 \\
9.7500 \\
9.6200\end{array}$ & $\begin{array}{l}3.57990 \\
3.72467 \\
3.59398 \\
3.65607\end{array}$ & $\begin{array}{l}0.35799 \\
0.37247 \\
0.35940 \\
0.21108\end{array}$ \\
\hline Precognition & $\begin{array}{l}\text { Muslim } \\
\text { Hindu } \\
\text { Christian } \\
\text { Total }\end{array}$ & $\begin{array}{l}100 \\
100 \\
100 \\
300\end{array}$ & $\begin{array}{l}9.7800 \\
11.8200 \\
10.4500 \\
10.6833\end{array}$ & $\begin{array}{l}3.47162 \\
4.17395 \\
3.66081 \\
3.86257\end{array}$ & $\begin{array}{l}0.34716 \\
0.41739 \\
0.36608 \\
0.22301\end{array}$ \\
\hline $\begin{array}{l}\text { Total paranormal } \\
\text { beliefs }\end{array}$ & $\begin{array}{l}\text { Muslim } \\
\text { Hindu } \\
\text { Christian } \\
\text { Total }\end{array}$ & $\begin{array}{l}100 \\
100 \\
100 \\
300 \\
\end{array}$ & $\begin{array}{l}75.7900 \\
77.5000 \\
77.4600 \\
76.9167 \\
\end{array}$ & $\begin{array}{l}11.99435 \\
14.46591 \\
11.89722 \\
12.82281 \\
\end{array}$ & $\begin{array}{l}1.19944 \\
1.44659 \\
1.18972 \\
0.74033 \\
\end{array}$ \\
\hline
\end{tabular}


Table 9. Summary of one-way analysis of variance for paranormal beliefs and its subscales with regard to religion. Number of groups $=3$.

\begin{tabular}{|c|c|c|c|c|c|c|}
\hline $\begin{array}{l}\text { Paranormal } \\
\text { beliefs }\end{array}$ & $\begin{array}{l}\text { Sources of } \\
\text { variance }\end{array}$ & $\begin{array}{l}\text { Sum of } \\
\text { squares }\end{array}$ & df & $\begin{array}{l}\text { Mean } \\
\text { square }\end{array}$ & $\mathbf{F}$ & $\mathbf{p}$ \\
\hline $\begin{array}{l}\text { Traditional } \\
\text { religious } \\
\text { beliefs }\end{array}$ & $\begin{array}{l}\text { Between groups } \\
\text { Within groups } \\
\text { Total }\end{array}$ & $\begin{array}{l}176.827 \\
2483.210 \\
2660.037\end{array}$ & $\begin{array}{l}2 \\
297 \\
299\end{array}$ & $\begin{array}{l}88.413 \\
8.361\end{array}$ & 10.575 & 0.0005 \\
\hline Psi & $\begin{array}{l}\text { Between groups } \\
\text { Within groups } \\
\text { Total }\end{array}$ & $\begin{array}{l}30.927 \\
2829.860 \\
2860.787\end{array}$ & $\begin{array}{l}2 \\
297 \\
299\end{array}$ & $\begin{array}{l}15.463 \\
9.528\end{array}$ & 1.623 & 0.199 \\
\hline Witchcraft & $\begin{array}{l}\text { Between groups } \\
\text { Within groups } \\
\text { Total }\end{array}$ & $\begin{array}{l}141.307 \\
3660.330 \\
3801.637\end{array}$ & $\begin{array}{l} \\
297 \\
299\end{array}$ & $\begin{array}{l}70.653 \\
12.324\end{array}$ & 5.733 & 0.004 \\
\hline Superstition & $\begin{array}{l}\text { Between groups } \\
\text { Within groups } \\
\text { Total }\end{array}$ & $\begin{array}{l}4.820 \\
1533.660 \\
1538.480\end{array}$ & $\begin{array}{l} \\
297 \\
299\end{array}$ & $\begin{array}{l}2.410 \\
5.164\end{array}$ & 0.467 & 0.628 \\
\hline Spiritualism & $\begin{array}{l}\text { Between groups } \\
\text { Within groups } \\
\text { Total }\end{array}$ & $\begin{array}{l}61.220 \\
3319.660 \\
3380.880\end{array}$ & $\begin{array}{l} \\
297 \\
299\end{array}$ & $\begin{array}{l}30.610 \\
11.177\end{array}$ & 2.739 & 0.066 \\
\hline $\begin{array}{l}\text { Extra- } \\
\text { ordinary life } \\
\text { forms }\end{array}$ & $\begin{array}{l}\text { Between groups } \\
\text { Within groups } \\
\text { Total }\end{array}$ & $\begin{array}{l}75.740 \\
3920.940 \\
3996.680\end{array}$ & $\begin{array}{l}2 \\
297 \\
299\end{array}$ & $\begin{array}{l}37.870 \\
13.202\end{array}$ & 2.869 & 0.058 \\
\hline Precognition & $\begin{array}{l}\text { Between groups } \\
\text { Within groups } \\
\text { Total }\end{array}$ & $\begin{array}{l}216.247 \\
4244.670 \\
4460.917\end{array}$ & $\begin{array}{l}2 \\
297 \\
299\end{array}$ & $\begin{array}{l}108.123 \\
14.292\end{array}$ & 7.565 & 0.001 \\
\hline $\begin{array}{l}\text { Total } \\
\text { paranormal } \\
\text { beliefs }\end{array}$ & $\begin{array}{l}\text { Between groups } \\
\text { Within groups } \\
\text { Total }\end{array}$ & $\begin{array}{l}190.487 \\
48972.430 \\
49162.917\end{array}$ & $\begin{array}{l}2 \\
297 \\
299\end{array}$ & $\begin{array}{l}95.243 \\
164.890\end{array}$ & 0.578 & 0.562 \\
\hline
\end{tabular}

As seen in table - 9, there is significant difference between the mean scores on TRB at least in two groups with regard to religiosity, $\quad(\mathrm{F} 2,297=10.575, \mathrm{p}<0.01)$. Also, there is significant difference between at least two groups on WC $(F$ 2,297 $=5.733, \mathrm{p}<0.01)$. The table also reports significant difference on $\mathrm{PR}$ at least between two groups with regard to religiosity (F 2,297=7.565, p <0.01).

For the clarification of these differences Tukey's Post-Hoc is used as follows: 
Table 10: Tukey’s Post-hoc in (TRB) with regard to Religiosity.

\begin{tabular}{|l|l|l|l|l|}
\hline Group(i) & Group(j) & Mean diff. (i-j) & Std.error & p \\
\hline Muslim & Hindu & 1.88 & 0.409 & 0.0005 \\
\hline
\end{tabular}

As seen in table- 10, Muslim subjects have higher scores on (TRB) in comparison with Hindu subjects.

Table 11: Tukey’s Post-hoc in (WC) with regard to Religiosity.

\begin{tabular}{|l|l|l|l|l|}
\hline Group(i) & Group(j) & Mean diff. (i-j) & Std.error & p \\
\hline Muslim & Hindu & 1.64 & 0.496 & 0.003 \\
\hline
\end{tabular}

As seen in table- 11, Muslim subjects have higher scores on (WC) in comparison with Hindu subjects.

Table 12: Tukey’s Post-hoc in (PR) with regard to Religiosity.

\begin{tabular}{|l|l|l|l|l|}
\hline Group(i) & Group(j) & Mean diff. (i-j) & Std.error & p \\
\hline Muslim & Hindu & -2.04 & 0.535 & 0.0005 \\
\hline
\end{tabular}

As seen in table-12, Hindu subjects have higher scores on (PR) in comparison with Muslim subjects.

For testing Hypothesis-5: Socially marginal groups will be more susceptible to paranormal beliefs, independent t-test is used as follows: 
Table 13 Descriptive statistics of the low and high socio-economic status individuals with regard to Paranormal belief and its subscales.

\begin{tabular}{|l|l|l|l|l|l|}
\hline Paranormal Beliefs & SES & N & Mean & SD & Std.error of mean \\
\hline $\begin{array}{l}\text { Traditional religious } \\
\text { beliefs (TRB) }\end{array}$ & Low & 37 & 13.7838 & 3.11033 & 0.51134 \\
& High & 263 & 11.9696 & 3.03023 & 0.18685 \\
\hline Psycho kinesis (Psi) & Low & 37 & 17.2432 & 1.87684 & 0.30855 \\
& High & 263 & 16.3308 & 3.09285 & 0.19071 \\
\hline Witchcraft (WC) & Low & 37 & 12.5946 & 3.27838 & 0.53896 \\
& High & 263 & 10.9772 & 3.56502 & 0.21983 \\
\hline ssSuperstition (ST) & Low & 37 & 8.1892 & 2.92345 & 0.48061 \\
& High & 263 & 6.0114 & 2.02745 & 0.12502 \\
\hline Spiritualism (SP) & Low & 37 & 12.6757 & 3.39161 & 0.55758 \\
& High & 263 & 10.2167 & 3.25192 & 0.20052 \\
\hline $\begin{array}{l}\text { Extraordinary life forms } \\
\text { (EOLF) }\end{array}$ & Low & 37 & 10.1351 & 3.62217 & 0.59548 \\
& High & 263 & 9.5475 & 3.66183 & 0.22580 \\
\hline Precognition (PR) & & & & & \\
\hline paranormal & Low & 37 & 88.1892 & 13.12512 & 2.15776 \\
& High & 263 & 75.3308 & 11.97934 & 0.73868 \\
\hline $\begin{array}{l}\text { Total } \\
\text { beliefs ( Total P) }\end{array}$ & & 263 & 10.2776 & 3.73386 & 0.23024 \\
\hline
\end{tabular}


Table- 14: Independent t-test showing a comparison between the subjects of high and low Socio-economic status on Paranormal beliefs.

\begin{tabular}{|l|l|l|l|l|l|}
\hline Paranormal Beliefs & $\mathbf{t}$ & $\mathbf{d f}$ & $\mathbf{P}$ & Mean diff. & Std.error of diff. \\
\hline $\begin{array}{l}\text { Traditional religious } \\
\text { beliefs }\end{array}$ & 1.748 & 298 & 0.081 & 0.91244 & 0.52192 \\
\hline Psi & 3.399 & 298 & 0.001 & 1.81420 & 0.53377 \\
\hline Witchcraft & 2.608 & 298 & 0.010 & 1.61741 & 0.62009 \\
\hline Superstition & 4.385 & 298 & 0.0005 & 2.17778 & 0.49661 \\
\hline Spiritualism & 4.150 & 298 & 0.0005 & 2.45895 & 0.59254 \\
\hline $\begin{array}{l}\text { Extraordinary } \\
\text { forms }\end{array}$ & 0.915 & 298 & 0.361 & 0.58761 & 0.64212 \\
\hline Precognition & 5.227 & 298 & 0.0005 & 3.29000 & 0.62942 \\
\hline Total P & 5.638 & 298 & 0.0005 & 12.85839 & 2.28069 \\
\hline
\end{tabular}

Table- 14 shows that subjects of a low socioeconomic status have greater scores on Psi in comparison with their high socioeconomic status counterparts with 99\% confidence, t (298) =3.399, $\mathrm{p}<0.01$. Also, subjects of a low socioeconomic status have scored higher than those of a high socioeconomic status on Witchcraft, $t(298)=2.608, \mathrm{p} \leq 0.01$. Hence there is significant difference between the two groups. The table also shows that there is significant difference between the two groups of subjects on Superstition subscale, t (298) $=4.385, \mathrm{p}<0.01$. Again, low socioeconomic status subjects have scored significantly higher than the high socioeconomic status subjects with 99\% confidence on the Spiritualism, $\mathrm{t}=4.150(298), \mathrm{p}<0.001$ and Precognition subscale, $\mathrm{t}(298)=5.227, \mathrm{p}<0.001$ as also the total paranormal belief scale, $\mathrm{t}(298)=$ $5.638, \mathrm{p}<0.01$. The table also reports significant differences between the two groups of subjects with 95\% confidence on the traditional religious belief subscale. However, it is seen that there is no significant difference between the two groups with regard to the extraordinary life forms subscale, $\mathrm{t}(298)=0.915, \mathrm{p}>0.05$.

For examining Hypothesis-6: Women will endorse greater global paranormal beliefs than men, independent t-test is used as follows: 
Table -15: Descriptive statistics of the males and females on Paranormal belief and its subscales.

\begin{tabular}{|c|c|c|c|c|c|}
\hline Paranormal Beliefs & Gender & $\mathbf{N}$ & Mean & SD & Std.error of mean \\
\hline $\begin{array}{l}\text { Traditional religious } \\
\text { beliefs (TRB) }\end{array}$ & $\begin{array}{l}\text { Female } \\
\text { Male }\end{array}$ & $\begin{array}{l}150 \\
150\end{array}$ & $\begin{array}{l}16.4733 \\
16.4133\end{array}$ & $\begin{array}{l}2.93257 \\
3.04152\end{array}$ & $\begin{array}{l}0.23944 \\
0.24834\end{array}$ \\
\hline $\begin{array}{c}\text { Psycho kinesis } \\
\text { (Psi) }\end{array}$ & $\begin{array}{l}\text { Female } \\
\text { Male }\end{array}$ & $\begin{array}{l}150 \\
150\end{array}$ & $\begin{array}{l}12.4000 \\
11.9867\end{array}$ & $\begin{array}{l}3.03005 \\
3.15162\end{array}$ & $\begin{array}{l}0.25733 \\
0.24740\end{array}$ \\
\hline Witchcraft (WC) & $\begin{array}{l}\text { Female } \\
\text { Male }\end{array}$ & $\begin{array}{l}150 \\
150\end{array}$ & $\begin{array}{l}11.6733 \\
10.6800\end{array}$ & $\begin{array}{l}3.69018 \\
3.37643\end{array}$ & $\begin{array}{l}0.30130 \\
0.27568\end{array}$ \\
\hline Superstition (ST) & $\begin{array}{l}\text { Female } \\
\text { Male }\end{array}$ & $\begin{array}{l}150 \\
150\end{array}$ & $\begin{array}{l}6.4933 \\
6.0667\end{array}$ & $\begin{array}{l}2.43230 \\
2.07790\end{array}$ & $\begin{array}{l}0.16966 \\
0.19860\end{array}$ \\
\hline Spiritualism (SP) & $\begin{array}{l}\text { Female } \\
\text { Male }\end{array}$ & $\begin{array}{l}150 \\
150\end{array}$ & $\begin{array}{l}10.9533 \\
10.0867\end{array}$ & $\begin{array}{l}3.41598 \\
3.26243\end{array}$ & $\begin{array}{l}0.26638 \\
0.27891\end{array}$ \\
\hline $\begin{array}{l}\text { Extraordinary life } \\
\text { forms (EOLF) }\end{array}$ & $\begin{array}{l}\text { Female } \\
\text { Male }\end{array}$ & $\begin{array}{l}150 \\
150\end{array}$ & $\begin{array}{l}9.9867 \\
9.2533\end{array}$ & $\begin{array}{l}3.53123 \\
3.75274\end{array}$ & $\begin{array}{l}0.28832 \\
0.30641\end{array}$ \\
\hline Precognition (PR) & $\begin{array}{l}\text { Female } \\
\text { Male }\end{array}$ & $\begin{array}{l}150 \\
150\end{array}$ & $\begin{array}{l}10.6933 \\
10.6733\end{array}$ & $\begin{array}{l}3.70913 \\
4.02258\end{array}$ & $\begin{array}{l}0.30285 \\
0.32844\end{array}$ \\
\hline $\begin{array}{l}\text { Total paranormal } \\
\text { beliefs (Total P) }\end{array}$ & $\begin{array}{l}\text { Female } \\
\text { Male }\end{array}$ & $\begin{array}{l}150 \\
150\end{array}$ & $\begin{array}{l}76.9667 \\
76.8667\end{array}$ & $\begin{array}{l}12.85824 \\
12.83016\end{array}$ & $\begin{array}{l}1.04987 \\
1.04758\end{array}$ \\
\hline
\end{tabular}


Table -16: Independent t-test showing comparison between males and females on Paranormal beliefs.

\begin{tabular}{|c|c|c|c|c|c|}
\hline $\begin{array}{l}\text { Paranormal } \\
\text { beliefs }\end{array}$ & $\mathbf{t}$ & df & $\mathbf{P}$ & Mean diff. & $\begin{array}{l}\text { Std.error of } \\
\text { diff. }\end{array}$ \\
\hline $\begin{array}{l}\text { Traditional } \\
\text { religious beliefs } \\
(\mathrm{TRB})\end{array}$ & 0.174 & 298 & 0.862 & 0.06000 & 0.34497 \\
\hline $\begin{array}{l}\text { Psycho kinesis } \\
\text { (Psi) }\end{array}$ & 1.158 & 298 & 0.248 & 0.41333 & 0.35697 \\
\hline Witchcraft (WC) & 2.432 & 298 & 0.016 & 0.99222 & 0.40839 \\
\hline Superstition (ST) & 1.633 & 298 & 0.103 & 0.42667 & 0.26120 \\
\hline Spiritualism (SP) & 2.247 & 298 & 0.025 & 0.86667 & 0.38567 \\
\hline $\begin{array}{l}\text { Extraordinary life } \\
\text { forms (EOLF) }\end{array}$ & 1.743 & 298 & 0.082 & 0.73333 & 0.42073 \\
\hline Precognition (PR) & 0.045 & 298 & 0.964 & 0.02000 & 0.44676 \\
\hline $\begin{array}{l}\text { Total paranormal } \\
\text { beliefs (Total P) }\end{array}$ & 0.067 & 298 & 0.946 & 0.10000 & 1.48312 \\
\hline
\end{tabular}

Table 16 shows that females have greater scores on witchcraft in comparison to males with 99\% confidence, $\mathrm{t}(298)=2.432$, $\mathrm{p} \leq 0.01)$. Also, on the spiritualism subscales females have scored higher than the males, $\mathrm{t}(298)=2.247, \mathrm{p}<0.05)$. Hence there is significant difference between the two groups. The table also shows that there is no significant difference between the two groups of subjects on TRB, $\mathrm{t}(298)=0.174, \mathrm{p}>0.01 ; \mathrm{ST}, \mathrm{t}(298)=1.633, \mathrm{p}>0.01 ; \mathrm{Psi}, \mathrm{t}(298)=$ 1.158, $\mathrm{p}>0.01$; EOLF, $\mathrm{t}(298)=1.743, \mathrm{p}>0.01)$ and PR subscales $\mathrm{t}(298)=0.045, \mathrm{p}>0.01)$.

For testing Hypothesis-7: There will be a higher degree of endorsement of the paranormal beliefs among the adults and elderly, One-way ANOVA is used as follows: 
Table- 17: Descriptive statistics of the three age groups of individuals on Paranormal beliefs.

\begin{tabular}{|c|c|c|c|c|c|}
\hline Paranormal beliefs & Groups & $\mathbf{N}$ & Mean & SD & $\begin{array}{l}\text { Std.error of } \\
\text { mean }\end{array}$ \\
\hline Traditional religious beliefs & $\begin{array}{l}\text { Young } \\
\text { Adult } \\
\text { Elderly } \\
\text { Total }\end{array}$ & $\begin{array}{l}101 \\
133 \\
66 \\
300\end{array}$ & $\begin{array}{l}16.4059 \\
16.6015 \\
16.1818 \\
16.4433\end{array}$ & $\begin{array}{l}2.94339 \\
3.05490 \\
2.91895 \\
2.98269\end{array}$ & $\begin{array}{l}0.29288 \\
0.26489 \\
0.35930 \\
0.17221\end{array}$ \\
\hline Psi & $\begin{array}{l}\text { Young } \\
\text { Adult } \\
\text { Elderly } \\
\text { Total }\end{array}$ & $\begin{array}{l}101 \\
133 \\
66 \\
300\end{array}$ & $\begin{array}{l}12.1683 \\
11.8120 \\
13.0000 \\
12.1933\end{array}$ & $\begin{array}{l}2.82513 \\
3.03802 \\
3.46854 \\
3.09319\end{array}$ & $\begin{array}{l}0.28111 \\
0.26343 \\
0.42695 \\
0.17859\end{array}$ \\
\hline Witchcraft & $\begin{array}{l}\text { Young } \\
\text { Adult } \\
\text { Elderly } \\
\text { Total }\end{array}$ & $\begin{array}{l}101 \\
133 \\
66 \\
300\end{array}$ & $\begin{array}{l}10.8020 \\
11.5489 \\
11.0000 \\
11.1767\end{array}$ & $\begin{array}{l}2.93946 \\
3.84248 \\
3.83105 \\
3.56574\end{array}$ & $\begin{array}{l}0.29249 \\
0.33319 \\
0.47157 \\
0.20587\end{array}$ \\
\hline Superstition & $\begin{array}{l}\text { Young } \\
\text { Adult } \\
\text { Elderly } \\
\text { Total }\end{array}$ & $\begin{array}{l}101 \\
133 \\
66 \\
300\end{array}$ & $\begin{array}{l}6.2277 \\
6.1429 \\
6.6364 \\
6.2800\end{array}$ & $\begin{array}{l}2.27983 \\
2.23316 \\
2.31807 \\
2.26835\end{array}$ & $\begin{array}{l}0.22685 \\
0.19364 \\
0.28533 \\
0.13096\end{array}$ \\
\hline Spiritualism & $\begin{array}{l}\text { Young } \\
\text { Adult } \\
\text { Elderly } \\
\text { Total }\end{array}$ & $\begin{array}{l}101 \\
133 \\
66 \\
300\end{array}$ & $\begin{array}{l}11.0594 \\
10.1805 \\
10.3788 \\
10.5200\end{array}$ & $\begin{array}{l}3.66011 \\
3.41084 \\
2.65889 \\
3.36263\end{array}$ & $\begin{array}{l}0.36419 \\
0.29576 \\
0.32729 \\
0.19414\end{array}$ \\
\hline Extraordinary life forms & $\begin{array}{l}\text { Young } \\
\text { Adult } \\
\text { Elderly } \\
\text { Total }\end{array}$ & $\begin{array}{l}101 \\
133 \\
66 \\
300\end{array}$ & $\begin{array}{l}9.0396 \\
9.7218 \\
10.3030 \\
9.6200\end{array}$ & $\begin{array}{l}3.49834 \\
3.82051 \\
3.46397 \\
3.65607\end{array}$ & $\begin{array}{l}0.34810 \\
0.33128 \\
0.42638 \\
0.21108\end{array}$ \\
\hline Precognition & $\begin{array}{l}\text { Young } \\
\text { Adult } \\
\text { Elderly } \\
\text { Total }\end{array}$ & $\begin{array}{l}101 \\
133 \\
66 \\
300\end{array}$ & $\begin{array}{l}10.3465 \\
10.4511 \\
11.6667 \\
10.6833\end{array}$ & $\begin{array}{l}4.30914 \\
3.68549 \\
3.34817 \\
3.86257\end{array}$ & $\begin{array}{l}0.42878 \\
0.31957 \\
0.41213 \\
0.22301\end{array}$ \\
\hline Total paranormal beliefs & $\begin{array}{l}\text { Young } \\
\text { Adult } \\
\text { Elderly } \\
\text { Total }\end{array}$ & $\begin{array}{l}101 \\
133 \\
66 \\
300\end{array}$ & $\begin{array}{l}76.0495 \\
76.4586 \\
79.1667 \\
76.9167\end{array}$ & $\begin{array}{l}11.65965 \\
13.42968 \\
13.19858 \\
12.82281\end{array}$ & $\begin{array}{l}1.16018 \\
1.16450 \\
1.62463 \\
0.74033\end{array}$ \\
\hline
\end{tabular}


Table- 18. Summary of One-way analysis of variance for Paranormal beliefs and its subscales with regard to age. Number of groups $=3$.

\begin{tabular}{|c|c|c|c|c|c|c|}
\hline $\begin{array}{l}\text { Paranormal } \\
\text { beliefs }\end{array}$ & $\begin{array}{l}\text { Sources of } \\
\text { variance }\end{array}$ & $\begin{array}{l}\text { Sum of } \\
\text { squares }\end{array}$ & df & $\begin{array}{l}\text { Mean } \\
\text { square }\end{array}$ & $\mathbf{F}$ & $\mathbf{p}$ \\
\hline $\begin{array}{l}\text { Traditional } \\
\text { religious } \\
\text { beliefs }\end{array}$ & $\begin{array}{l}\text { Between groups } \\
\text { Within groups } \\
\text { Total }\end{array}$ & $\begin{array}{l}7.982 \\
2652.054 \\
2660.037\end{array}$ & $\begin{array}{l}2 \\
297 \\
299\end{array}$ & $\begin{array}{l}3.991 \\
8.929\end{array}$ & 0.447 & 0.640 \\
\hline Psi & $\begin{array}{l}\text { Between groups } \\
\text { Within groups } \\
\text { Total }\end{array}$ & $\begin{array}{l}62.347 \\
2798.439 \\
2860.787\end{array}$ & $\begin{array}{l}2 \\
297 \\
299\end{array}$ & $\begin{array}{l}31.174 \\
9.422\end{array}$ & 3.308 & 0.038 \\
\hline Witchcraft & $\begin{array}{l}\text { Between groups } \\
\text { Within groups } \\
\text { Total }\end{array}$ & $\begin{array}{l}34.665 \\
3766.972 \\
3801.637\end{array}$ & $\begin{array}{l}2 \\
297 \\
299\end{array}$ & $\begin{array}{l}17.332 \\
12.683\end{array}$ & 1.367 & 0.257 \\
\hline Superstition & $\begin{array}{l}\text { Between groups } \\
\text { Within groups } \\
\text { Total }\end{array}$ & $\begin{array}{l}11.159 \\
1527.321 \\
1538.480\end{array}$ & $\begin{array}{l}2 \\
297 \\
299\end{array}$ & $\begin{array}{l}5.580 \\
5.142\end{array}$ & 1.085 & 0.339 \\
\hline Spiritualism & $\begin{array}{l}\text { Between groups } \\
\text { Within groups } \\
\text { Total }\end{array}$ & $\begin{array}{l}46.037 \\
3334.843 \\
3380.880\end{array}$ & $\begin{array}{l}2 \\
297 \\
299\end{array}$ & $\begin{array}{l}23.018 \\
11.228\end{array}$ & 2.050 & 0.131 \\
\hline $\begin{array}{l}\text { Extraordinar } \\
\text { y life forms }\end{array}$ & $\begin{array}{l}\text { Between groups } \\
\text { Within groups } \\
\text { Total }\end{array}$ & $\begin{array}{l}66.192 \\
3930.488 \\
3996.680\end{array}$ & $\begin{array}{l}2 \\
297 \\
299\end{array}$ & $\begin{array}{l}33.096 \\
13.234\end{array}$ & 2.501 & 0.084 \\
\hline Precognition & $\begin{array}{l}\text { Between groups } \\
\text { Within groups } \\
\text { Total }\end{array}$ & $\begin{array}{l}82.446 \\
s 4378.470 \\
4460.917\end{array}$ & $\begin{array}{l}2 \\
297 \\
299\end{array}$ & $\begin{array}{l}41.223 \\
14.742\end{array}$ & 2.796 & 0.063 \\
\hline $\begin{array}{l}\text { Total } \\
\text { paranormal } \\
\text { beliefs }\end{array}$ & $\begin{array}{l}\text { Between groups } \\
\text { Within groups } \\
\text { Total }\end{array}$ & $\begin{array}{l}437.975 \\
48724.942 \\
49162.917\end{array}$ & $\begin{array}{l}2 \\
297 \\
299\end{array}$ & $\begin{array}{l}218.987 \\
164.057\end{array}$ & 1.335 & 0.265 \\
\hline
\end{tabular}

As seen in the Table 18, on the Psi subscale ( $F$ 2,297= 3.308, $\mathrm{p}<0.05$ ) there is significant difference between the mean scores of at least two groups on Psi.

For the clarification of differences on this subscale, Tukey's Post hoc is used as follows: 
Table -19: Tukey’s Post hoc on Psi subscale.

\begin{tabular}{|l|l|l|l|l|}
\hline Group (i) & Group (j) & Mean.diff ( $\mathbf{i}-\mathbf{j})$ & Std.error & $\mathbf{p}$ \\
\hline Elderly & Adult & 1.188 & 0.462 & 0.029 \\
\hline
\end{tabular}

Table -19 reports that the elderly subjects have greater scores on Psi subscale in comparison to adult subjects with $95 \%$ confidence $(\mathrm{p}<0.05)$.

\section{DISCUSSION}

The present research investigated the personality correlates of religiosity and paranormal beliefs. The findings proved heterogeneous with a number of significant relations emerging. Each finding and possible implications is discussed in the following sections.

The researcher sought to investigate the relationship between the paranormal beliefs and religiosity. As seen in (table1), significant correlation was found between the two constructs. The findings of the present study suggest that there was a significant positive correlation between religiosity and paranormal beliefs as well as between religiosity and the traditional religious beliefs, psi and witchcraft subscales of the paranormal beliefs scale. The findings of the present research also report a negative correlation between religious beliefs and the spiritualism, extraordinary-life-forms and precognition subscales of the paranormal beliefs scale. The findings of the present research provide supporting evidence for the relationship between the two constructs that was initially suggested by Goode (2000). The results are also consistent with the findings of yet another study which reported a negative correlation between religiosity and beliefs in telepathy, precognition and psi (Hillstrom and Strachan, 2000).

The findings of the present research suggest that Neuroticism is the only personality factor significantly predicting paranormal beliefs (see table 4). These findings are therefore consistent with the previous researches (Thalbourne, Dunber and Delin, 1995). The results were however inconsistent with the research findings of (Lester and Monagham, 1995; Willging and Lester, 1997), which reported that Neuroticism did not correlate with paranormal beliefs. Also, the findings of the present research did not reveal any correlation between Extraversion and paranormal beliefs, which contrasts the research findings of (Thalbourne, 1981; Eysenck, 1967; Thalbourne and Haraldson, 1980). The results of the present research were however consistent with the findings of (Lester et al.,1987; Windholz and Diamant, 1974), which reported extraversion to be a correlate of paranormal beliefs.

The results of the present study further revealed that Neuroticism, Openness and Conscientiousness were significant predictors of religiosity (see table 7). These findings provide partial supportive evidence to the findings of (Francis, 1992a, 1992b, 1993; Francis and Katz, 1992; Francis and Pearson, 1993; Lewis and Joseph, 1994; Lewis and Maltby, 1995, 1996; 


\section{Correlation of the Religious and the Paranormal Beliefs to Personality}

Maltby, 1999a, 1999b; Saroglou, 2002) which reported Agreeableness and Conscientiousness to be significant predictors of religiosity.

The present study sought to find out, if there are any differences in the degree of endorsement of the paranormal beliefs across different religions. The findings suggest that Muslims scored higher on the traditional religious beliefs subscale in comparison with the Hindus (see table 10). On the other hand, among the Hindus a higher degree of endorsement of witch-craft was observed (see table 11). However, so far as the Christians are concerned no significant differences were observed either on global paranormal beliefs or any of the subscales of the paranormal beliefs. The investigator did not find any previous researches being conducted on the personality correlates of religiosity and paranormal beliefs across three religious groups. Hence the present research is the first substantial exploratory investigation of its type.

The present research also sought to find out, whether the socially marginal groups were more susceptible to paranormal beliefs. The results revealed that the subjects of a low socio-economic status are more susceptible to paranormal beliefs. They scored higher on psi, witchcraft, superstition, spiritualism, precognition as well as the total- paranormal beliefs scale (see table 14). These results therefore provide supporting evidence for the social-marginality hypothesis, according to which people more susceptible to paranormal beliefs are members of socially marginal groups, such as the poorly educated or the un-employed that possess characteristics or roles that rank low among dominant social values (Bainbridge, 1978; \& Wuthnow, 1976). The deprivation and alienation associated with marginal status in the society is held to encourage such appeal to magical and religious beliefs, presumably because these beliefs bring various compensations to the lives of their adherents.

A large body of previous researches has reported that the endorsement of most, but certainly not all paranormal beliefs is stronger among women than men (Irwin, 1985; McGarry and Newberry; 1981). The results of the present study revealed that women scored higher than men on the witch-craft and spiritualism (see table 16). These results were inconsistent with the findings of Tobacyk and Pirttila- Backman (1992), who reported the belief in witch-craft to be higher among men than women. The present study reported no gender differences on global paranormal beliefs as well as traditional religious beliefs, superstition, psi, extraordinary life forms and precognition subscales (see table 16). Hence the results of the present study were inconsistent with the previous researches, suggesting women to score higher on global paranormal beliefs (Clarke, 1991; Rice, 2003; Tobayck and Milford, 1983; Wolfradt, 1997).

Gender differences may however be attributed to cultural differences. It has been found, via “top-down purification”, that the revised paranormal belief scale can yield systematically biased results with respect to gender differences in paranormal beliefs.

The present study also sought to find out, if there was any difference in the degree of endorsement of the paranormal beliefs across different age-groups. The results reported no significant differences between the three groups of subjects either on the global paranormal beliefs or the traditional religious beliefs, witch-craft, superstition, spiritualism, extraordinary life 


\section{Correlation of the Religious and the Paranormal Beliefs to Personality}

forms and precognition subscales of the paranormal beliefs scale (see table 18). However significant differences were found between the elderly and the adult subjects on the psi subscale, with elderly subjects scoring higher than the adults (see table 19). Hence the findings of the present research were not supportive of the earlier researches which suggested that most of the paranormal beliefs, with the major exception of traditional religious beliefs appear to be stronger in adults than in elderly people (Emmons and Sobal, 1981). So far as the young participants are concerned no significant differences were found either on the global paranormal beliefs or on any of the seven subscales of the paranormal beliefs.

\section{CONCLUSIONS}

The present research investigation of the personality correlates of religiosity and paranormal beliefs showed that paranormal beliefs and religiosity are indeed associated, confirming previous researches that suggested some kind of a relationship between the two (Goode, 2000; Haraldsson, 1981). The results of the present study confirmed the hypothesis based on previous studies, stating that Neuroticism is correlated with paranormal beliefs (Thalbourne, Dunbar and Delin, 1995). However, this hypothesis was partly confirmed, the findings could not confirm the other half of this hypothesis that Extraversion is correlated to paranormal beliefs (Thalbourne, 1981; Eysenck, 1967; Thalbourne and Haraldsson, 1980). The present study reported that only Neuroticism is correlated with paranormal beliefs. With regard to the religiosity, the personality factors, Neuroticism, Openness and conscientiousness were found to be significantly correlated to religiosity. These findings extended previous studies indicating, Agreeableness and Conscientiousness to be associated with religiosity (Francis, 1992a, 1992b, 1993; Francis and Katz, 1992; Francis and Pearson, 1993; Lewis and Joseph, 1994; Lewis and Maltby, 1995, 1996; Maltby, 1999a, 1999b; Saroglou, 2002). With regards to the different religious groups, the results of the present study showed that there are higher endorsements of traditional religious beliefs and witch-craft among the Muslims, while as the Hindus endorse a higher degree of precognition. These findings were the results of the first exploratory study of its type; hence further research is required to confirm the findings of the present research. The results also showed that the individuals of low socio-economic status were highly susceptible to paranormal beliefs. The results of the present research therefore provided supporting evidence for the socialmarginality hypothesis, according to which people more susceptible to paranormal beliefs are members of socially marginal groups, such as the poorly educated or the un-employed that possess characteristics or roles that rank low among dominant social values ( Bainbridge, 1978) and Wuthnow, 1976). With regard to gender significant differences were found on witch-craft and spiritualism. However, no significant differences were found on the global paranormal beliefs, thus the results of the present research provide partial support to the findings of the previous researches that showed women to endorse greater global paranormal beliefs (Clark, 1991; Rice, 2003; Tobayck and Milford, 1983; Wolfradt, 1997). With regard to age the results of the present research revealed there were significant differences between the adults and the elderly on the psi subscale, the elderly scored higher than the adults. These findings were not supportive of the previous researches which suggested that most of the paranormal beliefs, with 


\section{Correlation of the Religious and the Paranormal Beliefs to Personality}

the major exception of the traditional religious beliefs appear to be stronger in adults than the elderly people (Emmons and Sobal, 1981).

\section{SUGGESTIONS FOR FUTURE RESEARCH}

The present study was conducted exclusively on Indians; although the sample was fairly representative of the demographic areas sampled, the racial homogeneity of the sample limited the generalizability of the findings to other populations. Cultural differences are also seen to influence the results by a great degree; hence it would be better if this study is conducted in a cross-cultural context. There are also certain doubts regarding the dimensionality of the Revised Paranormal Beliefs Scale; non linear research methods must therefore be used to capture the wholeness of the paranormal phenomena. It would also be relevant to take into account the phenomenon of spirituality for a more informed research. Finally, all measures employed the self-report method, as is characteristic of this research (Kane \& Kane, 2000). A multi-method approach would limit the shortcomings associated with any one assessment method, and could be considered for future research.

\section{REFERENCES}

Aarnio, Kia and Lindeman, Marjaana. (2006). Paranormal beliefs, education and thinking styles. Personality and individual differences, 39(7),1227-1236.

American Psychiatric Association (1994). Diagnostic and statistical manual of mental disorders ( $4^{\text {th }}$ Ed.). Washington DC: Author.

Blackmore, S. J. (1994). Are women more sheepish? Gender differences in belief in the paranormal. In L. Coley and R. A. White (Eds.), Women and parapsychology. New York: Parapsychology Foundation.

Boshier, R. (1973). Conservatism and superstitious behaviour. In G. D. Wilson (Ed.), The psychology of conservatism, London: Academic Press.

Bourke, Rosamund and Francis, Leslie, J. (2000). Personality and religion among music students. Pastoral psychology. 48(6), 437-444.

Broad, C. D. (1949). The relevance of psychical research to philosophy. Philosophy, 24, 291309.

Buss, D. M. (1989). Personality as traits. American Psychologist, 44,1378-1388.

Caspi, A. (1998). Personality development across the life course. In W. Damon (Ed.), Handbook of child psychology. Volume 3: social, emotional, and personality development (pp. 311-388). New York: Wiley.

Chau, L. L., Johnson, R. C., Bowers, J. K., Darvill, T. J. \& Danko, G. P. (1990). Intrinsic and extrinsic religiosity as related to conscience, adjustment, and altruism. Personality and Individual Differences, 11, 397-400. 


\section{Correlation of the Religious and the Paranormal Beliefs to Personality}

Chequers, J., Joseph, S. \& Diduca, D. (1997). Belief in extraterrestrial life, UFO-related beliefs, and schizotypal personality. Personality and Individual Differences, 23, 519-521.

Clark, D. (1996). Experience and other reasons given for belief and disbelief in paranormal and religious phenomena. Journal of the society for psychical research. 60(841), 371-384.

Clarke, D. (1991). Belief in the paranormal: a New Zealand survey. Journal of the Society for Psychical Research, 57, 412-425.

Costa, P. T., Jr., \& McCrae, R. R. (1978). Objective personality assessment. In M. Storandt, I. C. Siegler, and M. F. Elias (Eds.), The clinical psychology of Ageing (pp. 119-143). New York: Plenum.

Costa, P. T., Jr., \& McCrae, R. R. (1992). Revised NEO Personality Inventory (NEO-PI-R) and the NEO Five Factor Inventory (NEO-FFI) professional manual. Odessa, FL: Psychological Assessment Resources.

Costa, P. T., Jr., \& McCrae, R. R. (1995). Primary traits of Eysenck’s P-E-N system: Three- and five-factor solutions. Journal of Personality and Social Psychology, 69, 308-317.

D’Onofrio, B. M., Eaves, L. J., Murrelle, L., Maes, H. H. \& Spilka, B. (1999). Understanding biological and social influences on religious affiliation, attitudes, and behaviours: a behaviour genetic perspective. Journal of Personality, 67, 953-984.

Davies, M. F. \& Kirkby, H. E. (1985). Multidimensionality of the relationship between perceived control and belief in the paranormal: spheres of control and types of paranormal phenomena. Personality and Individual Differences, 6, 661-663.

Desimpelaere, P., Sulas, F., Duriez, B., \& Hutsebaut, D. (1999). Psycho-epistemological styles and religious beliefs. The International Journal for the Psychology of Religion, 9, 125137.

Digman, J. M. (1997). Higher-order factors of the Big Five. Journal of Personality and Social Psychology, 73, 1246-1256.

Dudley, R. Thomas. (2000). Social acceptability of paranormal and religious beliefs. Psychological reports. 85(1), 255-256.

Dunne, Michael, P., Martin, Nicholas, G., Pangan, Theresa and Heath, Andrew, C. (1997). Personality and change in the frequency of religious observance. Personality and individual differences. 23(3), 527-530.

Duriez, B. \& Hutsebaut, D. (2000). The relation between religion and racism: the role of post critical beliefs. Mental Health, Religion and Culture, 3, 85-102.

Duriez, B. (2002a). Vivisecting the religious mind. Religiosity and need for closure. Manuscript submitted for publication.

Duriez, B. (2002b). People are strange when you're a stranger. A research note on the relation between religiosity and racism. Manuscript submitted for publication. 


\section{Correlation of the Religious and the Paranormal Beliefs to Personality}

Duriez, B., Fontaine, J.R.J. \& Hutsebaut, D. (2000). A further elaboration of the Post-Critical Belief scale: evidence for the existence of four different approaches to religion in Flanders - Belgium. Psychologica Beligica, 40, 153-181.

Duriez, B., Luyten, P., Snauwaert, B. \& Hutsebaut, D. (2002). The relative importance of religiosity and values in predicting political attitudes. Evidence for the continuing importance of religion in Flanders (Belgium). Mental Health, Religion and Culture, 5, 35-54.

Duriez, B., Soenens, B. \& Beyers, W. (2004). Personality, identity styles, and religiosity: an integrative study among late adolescents in Flanders (Belgium). Journal of Personality, 72 (5), 877-908.

Eckblad, M. \& Chapman, L. J. (1983). Magical ideation as an indicator of schizotypy. Journal of Counselling and Clinical Psychology, 51, 215-225.

Eliade, M. (Ed.) (1990). The encyclopaedia of religion. London: MacMillan Publishing Co.

Emme, E. E. (1940). Modification and origin of certain beliefs in superstition among 96 college students. Journal of Psychology, 10, 279-291.

Eysenck, H. (1967). Personality and extrasensory perception. Journal of the Society for Psychical Research, 44, 55-71.

Eysenck, H. J. \& Eysenck, S. B. (1968). A factorial study of psychoticism as a dimension of personality. Multivariate Behavioural Research, Special Issues, 15-31.

Eysenck, H.J. \& Eysenck, M.W. (1985). Personality and individual differences: a natural science approach. New York: Plenum.

Eysenck, M. W. (1998). Personality and the psychology of religion. Mental Health, Religion and Culture, 1, 11-19.

Farias, Miguel, Claridge, Gordon and Laufee, Maansur.(2006). Personality and cognitive predictors of the New practices and beliefs. Personality and individual differences. 39(5), 979-989.

Flori, K, L., Brown, E, E., Cortina, K, S. and Antonucci, Toni, C. (2006). "Locus of control as a mediator of the relationship between religiosity and life satisfaction: age, race, and gender differences."Erratum. Mental health, religion and culture. 9(4), 409.

Fontaine, J. R. J., Duriez, B., Luyten, P. \& Hutsebaut, D. (2003). The internal structure of the Post-Critical Belief scale. Personality and Individual Differences, 35, 501-518.

Francis, L, J., Lewis, J, M., Brown, L, B., Philip chalk, Ronald et al. (1996).

Francis, L. J. \& Katz, Y. J. (1992). The relationship between personality and religiosity in an Israeli sample. Journal for the Scientific Study of Religion, 31, 153-162. 


\section{Correlation of the Religious and the Paranormal Beliefs to Personality}

Francis, L. J. \& Pearson, P. R. (1993). The personality characteristics of student churchgoers. Personality and Individual Differences, 15, 373-380.

Francis, L. J. (1992a). Is psychoticism really a dimension of personality fundamental to religiosity? Personality and Individual Differences, 13, 645-652.

Francis, L. J. (1992b). Religion, neuroticism, and psychoticism. In J. F. Schumaker (Ed.), Religion and mental health (pp. 149-160). New York: Oxford University Press.

Francis, L. J. (1993). Personality and religion among college students in the U.K. Personality and Individual Differences, 14, 619-622.

Francis, Leslie, J. and Jackson, Chris, J. (2003). Eysenck's dimensional model of personality and religion: are religious people more neurotic? Mental health, religion and culture. 6(1), 87-10.

Francis, Leslie, J. and Jones, Susan, H. (1999). Personality and Christian beliefs among adult church- goers. Journal of psychological type. 47, 5-11.

French, C. (1992). Factors underlying belief in the paranormal: do sheep and goats think differently? Bulletin of the British Psychological Society, 5, 295-299.

Gallup, G. Jr. \& Newport, F. (1991). Belief in paranormal phenomena among adult Americans. Skeptical Enquirer, 15, 137-146.

Gallup, G. Jr., (1997). Public Opinion 1996. Wilmington, DE: Scholarly Resources.

Goode, E. (2000). Two paranormalisms or two and half? An empirical exploration. Skeptical Enquirer, 24 (1), 29-35.

Gow, Kathryrn, Lang, Tracey and Chant, David. (2005). Fantasy proneness, paranormal beliefs and personality features in out-of-body experiences. Contemporary Hypnosis. 21(3), 107125.

Greene, F, Gordon. (1999). A projection geometry for separation experiences. Journal of NearDeath studies. 17(3), 151-191.

Groth- Marnat, Gary and Pegden, Julie- Ann.(1998). Personality correlates of paranormal beliefs: Locus of control and sensation seeking. Social behavior and personality. 26(3), 291-296.

Haraldson, Erlender and Houtkooper, Joop, M. (1997). Traditional Christian beliefs, spiritualism and the paranormal: An Icelandic-American comparison. International journal for the psychology of religion. 6(1), 51-64.

Harvey, J. Irwin. (1993). Belief in the Paranormal: A Review of the Empirical Literature. The Journal of the American Society For Psychical Research ,1, 83.

Heaven, P. C. (1990). Religious values and personality dimensions. Personality and Individual Difference, 11, 953-956. 


\section{Correlation of the Religious and the Paranormal Beliefs to Personality}

Hills, Peter, Francis, Leslie, J.,Argyle, Michael and Jackson, Chris, J.(2004). Primary personality trait correlates of religious practice and orientation. Personality and individual differences. 36(1), 61-73.

Hillstrom, E. L. \& Strachan, M. (2000). Strong commitment to traditional Protestant religious beliefs is negatively related to beliefs in paranormal phenomena. Psychological Reports, 86, 183-189.

Houran, James. (1997). Preliminary study of death anxiety of believers versus percipients of the paranormal. Psychological reports. 80(1), 345-346.

Hutsebaut, D. (1996). Post-critical belief: a new approach to the religious attitude problem. Journal of Empirical Theology, 9 (2), 48-66.

Hutsebaut, D. (1999). Cursus Godsdienstpsychologie. Cursusdiens Psychologische Kring. Unpublished manuscript: K. U. Leuven.

Hutsebaut, D. (2000). Post-Critical Belief scales. Exploration of a possible developmental process. Journal of Empirical Theology, 13(2), 19-28.

Irwin, H. J. (1990). Fantasy proneness and paranormal beliefs. Psychological Reports, 66, 655658.

Irwin, H. J. (1993). Belief in the paranormal: a review of empirical literature. Journal of the Society for Psychical Research, 87, 1-39.

Irwin, H. J. (1994). Paranormal beliefs and proneness to dissociation. Psychological Reports, 75, 1344-1346.

Jorm, Anthony, F. and Christensen, Helen. (2004). Religiosity and personality: Evidence for non- linear association. Personality and individual differences. 36(6), 1433-1441.

Kia, A. and Marjaana, L. (2007). Religious people and paranormal believers: Alike or Different? Journal of individual difference, 28 (1), 1-9.

Knox, David, Langehough, Steven, O, Walters, Connor and Rowley, Michael. (1999). Religiosity and spirituality among college students. College student journal. 32(3), 430432.

Kosek, R. B. (1999). Adaptation of the Big Five as a hermeneutic instrument for religious constructs. Personality and Individual Differences, 27, 229-237.

Kosek, R. B. (2000). The desire for God: an assessment of seminarians’ spirituality through the lens of the "Big Five”. Pastoral Psychology, 49, 43-50.

Langer, E. J. (1975). The illusion of control. Journal of Personality and Social Psychology, 32, 311-328.

Lawrence, T. R. (1995). How many factors of paranormal belief are there? A critique of the Paranormal Belief Scale. Journal of Parapsychology, 59, 3-25. 


\section{Correlation of the Religious and the Paranormal Beliefs to Personality}

Lester, D. \& Monoghan, K. (1995). Belief in the paranormal and personality. Perceptual and Motor Skills, 81, 114.

Lester, D., Thinschmidt, J. \& Trautman, L. (1987). Paranormal belief and Jungian dimensions of personality. Psychological Reports, 61, 182.

Lewis, C. A. \& Joseph, S. (1994). Religiosity: psychoticism and obsessionality in Northern Irish university students. Personality and Individual Differences, 17, 685-687.

Lewis, C. A. \& Maltby, J. (1995). Religiosity and personality among U. S. adults. Personality and Individual Differences, 18, 293-295.

Lewis, C. A. \& Maltby, J. (1996). Personality, prayer, and church attendance in a sample of male college students in the USA. Psychological Reports, 78, 976-978.

Lewis, Christopher Alan, Francis, Leslie, J. and Enger, Trond. (2006). Personality, prayer and church-attendance among a sample of 11 to 18 year olds in Norway. Mental health, Religion and Culture. 7(3), 269-274.

Lucadou, Walter, V. (2001). Hans in luck: The currency of evidence in parapsychology. Journal of parapsychology. 65(1), 3-16.

Lukey, Nicole and Baruss, Imants. (2006). Intelligence correlates of transcendent beliefs: A preliminary study. Imagination, cognition and personality. 24(3),259-270.

Lundeen, G.E., \& Caldwell,O.W. (1930). A study of unfounded beliefs among high-school seniors. Journal of Educational Research, 22, 257-273.

Malinowski, B. (1948). Magic, science and religion. New York: Doubleday.

Maltby, J. (1999a). Religious orientation and Eysenck's personality dimensions: the use of the amended religious orientation scale to examine the relationship between religiosity, psychoticism, neuroticism and extraversion. Personality and Individual Differences, 26, 79-84.

Maltby, J. (1999b). Personality dimensions of religious orientation. Journal of Psychology, 133, 631-640.

Maltby, J.(1998). Personality correlates of religiosity among adults in the Republic of Ireland. Psychological Reports. 81(3, pt 1), 827-831.

Maria, E., Aguilar, V., Mahnaz, Moghauloo. (2007). Domain \& facet personality correlates of religiosity among Iranian college students. Mental health, Religion \& culture,23(1).

Mc Creery, Charles and Claridge, Gordon. (1996). Out- of- the- body experiences and personality. Journal of the society for psychical research. 60(838), 129-148.

McCrae, R. R. \& Costa, P. T., Jr. (1997). Conceptions and correlates of Openness to Experience. In R. Hogan, J. A. Johnson \& S. R. Briggs (Eds.), Handbook of personality psychology (pp. 269-290). Orlando, FL: Academic Press. 


\section{Correlation of the Religious and the Paranormal Beliefs to Personality}

McCrae, R. R. (1996a). Social consequence of experiential openness. Psychological Bulletin, 120, 323-337.

McCrae, R. R. (1996b). Towards a new generation of personality theories: theoretical contexts for the five-factor model. In J. S. Wiggins (Ed.), The five-factor model of personality: theoretical perspectives (pp. 51-87). New York: Guildford Press.

McCrae, R. R. (1999). Mainstream personality psychology and the study

McCrae, R. R., Zonderman, A. B., Costa, P. T., Jr., \& Bond, M. H. (1996). Evaluating replicability of factors in the revised NEO Personality Inventory: confirmatory analysis versus Procustes rotation. Journal of Personality and Social Psychology, 70, 552-566.

McGarry, J. J. \& Newberry, B. H. (1981). Beliefs in paranormal phenomena and locus of control: a field study. Journal of Personality and Social Psychology, 41, 725-736.

Messer, W. S. \& Griggs, R. A. (1989).Student belief and involvement in the paranormal and performance in introductory psychology. Teaching of Psychology, 16, 187-191.

Michel, E. McCullough, Tsang, J.A, \& Sharion, B. (2003). Personality traits in adolescents as predictors of religiousness in early adulthood: Findings from the Terman longitudinal study. Personality and Social Psychology Bulletin, 29; 980.

Musgrave, Cassandra. (1998). The Near-death experience: A study of spiritual transformation. Journal of Near-Death studies.15(3), 187-201.

Norenzayan, Ara and H ansen, Ian, G. (2006). Beliefs in the supernatural agents in the face of Death. Personality and social psychological Bulletin. 32(2), 174-187.

of religion. Journal of Personality, 67, 1208-1212.

Okebukola, P. A. (1986). Relationship between anxiety, belief system, and creativity. Journal of Social Psychology, 126, 815-816.

Pekala, R.J., Kumar, V.K. \& Marcano, G. (1995). Anomalous paranormal experiences, hypnotic-susceptibility, and dissociation. Journal of the Society for Physical Research, 89, 313-332.

Peltzer, Karl. (2002). Paranormal beliefs and personality among black south African students. Social behavior and personality. 30 (4), 391-398.

Personality and religion among undergraduate students in the united kingdom, united states, Australia, and Canada. Journal of psychology and Christianity. 14(3), 250-262.

Peter, H. and Paul, R. (2007-2008). Individuals who report themselves to be abducted by Aliens: investigating the differences in Fantasy proneness, Emotional intelligence and the BigFive personality factors. Imagination, cognition and personality. 27(2), 139-161.

Phillip, J. R. (1996). Inquiry into the Paranormal. Nursing Science Quarterly, 9;89. 


\section{Correlation of the Religious and the Paranormal Beliefs to Personality}

Rattet, S. L. \& Bursik, K. (2001). Investigating the personality correlates of paranormal belief and pre-cognitive experience. Personality and Individual Differences, 31, 433-444.

Rice, T. W. (2003). Believe it or not: religious and other paranormal beliefs in the United States. Journal for the Scientific Study of Religion, 42, 95-106.

Ricoeur, P. (1970). Symbolen van het kwaad. Rotterdam: Lemniscaat. Roberts, B. W., Caspi, A. \& Moffitt, T. E. (2001). The kids are alright: growth and stability in personality development from adolescence to adulthood. Journal of Personality and Social Psychology, 81, 670-683.

Robinson, T. N. (1990). Eysenck personality measures and religious orientation. Personality and Individual Differences, 11, 915-921.

Roig, M., Bridges, K. R., Renner, C. H. \& Jackson, C. R. (1998). Belief in the paranormal and its association with irrational thinking controlled for context effects. Personality and Individual Differences, 24 (2), 229-236.

Rudski, Jeffrey. (2004). What does a superstitious person believe? Impressions of participants. Journal of general psychology. 130(4), 431-445.

Saroglou, V. (2002). Religion and the five factors of personality: a meta-analytic review. Personality and Individual Differences, 32, 15-25.

Saucier, G. \& Goldberg, L. R. (1998). What is beyond the Big Five? Journal of Personality, 66, 495-524.

Saucier, G. (2000). Isms and the structure of social attitudes. Journal of Personality and Social Psychology, 78, 366-385.

Schmeidler, G. R. (1945). Separating the sheep from the goats. Journal of the American Society for Psychical Research, 39, 47-49.

Schouten, S. A. (1983). Attitude about technology and belief in ESP. Psychological Reports, 53, 358.

Schriever, F. (2000). Are there different cognitive structures behind paranormal beliefs? European Journal of Parapsychology, 15, 46-47.

Sebastian, K.A. and Mathew, V. Goerge.(2004). Personality correlates of Psi beliefs. Journal of Indian psychology. 21(2), 61-66.

Shutle, James, W. and Hosch, Harmon, M. (1996). Optimism, religiosity and neuroticism: A cross-cultural study. Personality and individual differences. 20(2), 239-244.

Sobal, J., \& Emmons, C.F. (1982). Patterns of belief in religious, psychic, and other paranormal phenomena. Zetetic Scholar, 9,7-17. 


\section{Correlation of the Religious and the Paranormal Beliefs to Personality}

Streyffeler, L. L. \& McNally, R. J. (1998). Fundamentalists and liberals: personality characteristics of Protestant Christians. Personality and Individual Differences, 24, 579580.

Stuart-Hamilton, Ian, Nayak, Laxman and Priest, Lee.(2006). Intelligence, beliefs in the paranormal, knowledge of personality and aging. Educational Gerontology. 32(3), 173184.

Taylor, A. \& McDonald, D. A. (1999). Religion and the five-factor model of personality: an exploratory investigation using a Canadian university sample. Personality and Individual Differences, 27, 1243-1259.

Ter Keurst, A.J. (1939). Comparative differences between superstitious and non- superstitious children. Journal of Experimental Education, 7,261-267.

Thalbourne, M. A. \& French, C. C. (1995). Paranormal belief, manic-depressiveness, and magical ideation: a replication. Personality and Individual Differences, 18, 291-292.

Thalbourne, M. A. \& Haraldsson, E. (1980). Personality characteristics and sheep and goats. Personality and Individual Differences, 1, 180-185.

Thalbourne, M. A. (1981). Extraversion and the sheep-goat variable: a conceptual replication. Journal of the American Society for Psychical Research, 75, 105-119.

Thalbourne, M. A. (1994). Belief in the paranormal and its relationship to schizophrenia-related measures: a confirmatory study. British Journal of Clinical Psychology, 33, 78-80.

Thalbourne, M. A. (1997). Paranormal belief and superstition - how large is the association? Journal of the American Society for Psychical Research, 91, 221-226.

Thalbourne, M. A. (2007). Potential psychological predictors of religiosity. International journal of psychology of religion. 17(4), 333-336.

Thalbourne, M. A., Dunbar, K. A. \& Delin, P. S. (1995). An investigation into correlates of belief in the paranormal. Journal of the Society for Physical Research, 89, 215-231.

Thalbourne, Michael, A. (1996). Further studies of the measurement and correlates of beliefs in the paranormal. Journal of the American society for psychic research. 89(3), 233-247.

Thalbourne, Michael, A. (1999). Personality characteristics of students who believe themselves to be psychic. Journal of the society for psychical research. 63(856), 203- 212.

Thalbourne, Michael, A. and Delin, Peter, S. (1999). Transliminality: its relation to dream life, religiosity and mystical experience. International journal for the psychology of religion. 9(1), 45-61.

Thalbourne, Michael, A. and Houtkooper, Joop, M. (2002). Reliogiosity/ Spirituality and beliefs in the paranormal: A German replication. Journal of the society for psychical research. 66(867), 113-115. 


\section{Correlation of the Religious and the Paranormal Beliefs to Personality}

Thalbourne, Michael, A. Dunbar, Kelly, A. and Delin, Peter, S. (1996). An investigation into correlates of beliefs in the paranormal. Journal of the American society for psychical research. 89(3), 215-231.

Thorson, J, A., Powell, F.C., Abdel- Khalek, A, M. and Beshai, J, A.(1998). Constructions of religiosity and death anxiety in two cultures: the united states and Kuwait. Journal of psychology and theology. 25(3), 374-383.

Tobacyk, J. J. (1982). Paranormal belief and trait anxiety. Psychological Reports, 51, 861-862.

Tobayck, J. \& Milford, G. (1983). Belief in paranormal phenomena: assessment instrument development and implications for personality functioning. Journal of Personality and Social Psychology, 44, 1029-1037.

Tobayck, J. J. \& Pirttilla-Backman, A. M. (1992). Paranormal beliefs and there implications in university students from Finland and the United States. Journal of Cross-Cultural Psychology, 23, 59-71.

Tobayck, J. J. (1995). Final thoughts on issues in the measurement of paranormal beliefs. The Journal of Parapsychology, 59, 141-145.

Truzzi, M. (1972). The occult revival as popular culture: Some random o bservations on the old and nouveau witch. The sociological Quaterly, 13, 16-36.

Verhoeven, D.\& Hutsebaut, D. (1995). Identity statuses and religiosity. A research among Flemish university students. Journal of Empirical Theology, 8(1), 46-64.

Vitulli, William, F. (1998). Beliefs in parapsychological events or experiences among college students in a course in experimental parapsychology. Perceptual and motor skills. 85(1), 273-274.

Wagner, M. W. \& Ratzeburg, F. H. (1987). Hypnotic suggestibility and paranormal belief. Psychological Reports, 60, 1069-1070.

Wallace, M. I. (1990). The second naiveté in Barth and Ricoeur. In M. I. Wallace (Ed.), The second naiveté: Barth, Ricoeur and the New Yale Theology (pp. 51- 87). Macon, GA. : Mercer University Press.

White, Josphine, Joseph, Stephen and Neil, Alastair. (1996). Religiosity, psychoticism and Schizotypal traits. Personality and individual differences. 19(6), 847-851.

Wilde, Alex and Joseph, Stephen.(1998). Religiosity and personality in a Moslem context. Personality and individual differences. 23(5), 899-900.

Willging, Brian, J. and Lester, David. (1998). Paranormal beliefs and personality scores of high school students. Perceptual and motor skills. 85(3, pt 1), 938.

Willing, B. T. \& Lester, D. (1997). Paranormal beliefs and personality scores of high school students. Perceptual and Motor Skills, 85, 938. 
Windholz, G. \& Diamant, L. (1974). Some personality traits of believers in extraordinary phenomena. Bulletin of the Psychonomic Society, 3, 125-126.

Wolfradt, U. (1997). Dissociative experiences, trait anxiety and paranormal beliefs. Personality and Individual Differences, 23, 15-19.

Wulff, D. M. (1991). Psychology of religion: classic and contemporary views. New York: Wiley.

Wulff, D. M. (1997). Psychology of religion: classic and contemporary views. New York: Wiley.

Wuthnow, R. (1976). Astrology and marginality. Journal of the Scientific Study of Religion, 15,157-168.

Zusne, L. , \& Jones, W.H. (1982). Anomalistic Psychology: A Study of Extraordinary phenomena of behavior and experience. Hillsdale, NJ: Lawrence Earlbaum. 